\title{
Regression of A549 lung cancer tumors by anti-miR-150 vector
}

\author{
YOU-JIE LI $^{1 *}$, YAN-XIA ZHANG ${ }^{1 *}$, PING-YU WANG ${ }^{1}$, YONG-LIANG CHI ${ }^{2}$, \\ CHAO ZHANG $^{1}$, YING MA ${ }^{1}$, CHANG-JUN LV ${ }^{3}$ and SHU-YANG XIE ${ }^{1}$ \\ ${ }^{1}$ Institute of Medical Molecular Genetics, Department of Biochemistry and Molecular Biology, \\ Binzhou Medical University, Yan Tai 264003; ${ }^{2}$ Shandong China Traditional Medical Affiliated Hospital, \\ Ji Nan 250012; ${ }^{3}$ The Affiliated Hospital to Binzhou Medical University, Binzhou 256603, P.R. China
}

Received July 25, 2011; Accepted August 23, 2011

DOI: 10.3892/or.2011.1466

\begin{abstract}
RNAs (miRNAs) have been shown to play a role in cancer. Antisense oligonucleotides can bind directly to miRNAs and block their activity, which are generally named anti-miRNAs. To suppress A549 cell proliferation in vitro and in vivo by anti-miRNAs, an anti-miR-150 expression vector (PR-ASO-150), regulated by the H1 promoter and containing a 'TTTTT' sequence following a hairpin to stop transcription, was constructed. A549 cell proliferation in vitro or in nude mice was observed after PR-ASO-150 treatment. Our results showed that miR-150 expression was inhibited and the growth inhibition rate of A549 cells was higher in the PR-ASO-150treated group compared with the control, which indicated that PR-ASO-150 could inhibit A549 cell proliferation by regulating miR-150 expression. Following establishment of A549 cancer cell xenografts in nude mice, PR-ASO-150 was delivered intratumorally to investigate the suppressive action to tumor proliferation by regulating miR-150 expression. The results indicate that the tumor volume and weight were lower compared to the control group. Our results further showed that p53 expression was higher after tumor tissue was treated with PR-ASO-150, indicating that up-regulation of p53 contributed
\end{abstract}

Correspondence to: Dr Shu-Yang Xie, Institute of Medical Molecular Genetics, Department of Biochemistry and Molecular Biology, Binzhou Medical University, 346 Guan Hai Road, Lai Shan District, Yan Tai 264003, P.R. China

E-mail: shuyangxie@yahoo.com.cn

Dr Chang-Jun Lv, The Affiliated Hospital to Binzhou Medical University, Binzhou 256603, P.R. China

E-mail: lucky_lcj@sina.com

${ }^{*}$ Contributed equally

Abbreviations: miRNAs, microRNAs; PR-ASO-150, anti-miR150 expression vector; AMOs, anti-microRNA oligonucleotides; MTT, 3-(4,5-dimethylthiazol-2-yl)-2,5-diphenyltetrazolium bromide; DMSO, dimethyl sulfoxide

Key words: antisense oligonucleotides, microRNAs, cell proliferation, cancer therapy to the suppression to tumor growth. Our study provides a novel strategy for cancer therapy through the development of antimiRNAs.

\section{Introduction}

microRNAs (miRNAs) are small non-coding RNA molecules (19-22 nucleotides) that bind to mRNA in a sequence-specific manner (1). Through complementary binding to mRNA targets, each microRNA has the distinct capability to potentially regulate the expression of hundreds of genes and thereby modulates several cellular pathways including proliferation and apoptosis (2).

Importantly, miRNAs play critical roles in the development and progression of several types of cancers $(3,4)$. Dependent upon the nature of their target gene(s), miRNAs may function as tumor suppressors by down-regulating target oncogenes (e.g. let-7 and miR-15/16) or as oncogenes by negatively controlling genes that regulate tumor cell differentiation and apoptosis (e.g. miR-155 and miR-21) (5). Indeed, several miRNAs are reported to have important roles in different types of cancer, including acute myelogenous leukemia $(6,7)$, chronic myelogenous leukemia (CML) (8-10), lung cancer $(11,12)$, and breast cancer (13), among others. miRNAs have also been shown to play a role in cancer progression through the modulation of cellular adhesion, cell matrix and signaling activities $(14,15)$. In addition, miRNAs have been shown to regulate the expression of hypoxia-related genes and of the vascular endothelial growth factor $(16,17)$.

Emerging evidence shows that deregulation of miRNAs may be a primary driver of cancer initiation and progression. The rules of Watson and Crick base-pairing guide the binding of miRNAs to their target genes. In order to circumvent this interaction, anti-microRNA oligonucleotides (AMOs) have been generated to directly compete with endogenous miRNAs (18). Several modifications of AMOs have been used to improve their effectiveness and stability such as the addition of 2'-O-methyl and 2'-O-methoxyethyl groups to the 5' end of the molecule (19). AMOs conjugated to antagomirs have also been generated and have been described to inhibit microRNA activity in vivo efficiently (20). miR-21, miR-155, and miR-17-5p are proven oncogenic miRNAs overexpressed in several solid cancers $(21,22)$. Lu et al designed three regular AMOs 
(AMO-21, AMO-155 and AMO-17) antisense to these miRNAs which produced significant decreases in cancer cell survival (23). AMOs specific to miR-21, and miR-181a have also been shown to inhibit A549 cell growth by inducing apoptosis and S-phase arrest (24). In a previous study, we demostrated that miR-20, miR-106, and miR-150 acted as oncogenic miRNAs and inhibition of the expression of these miRNAs by AMOs could suppress A549 cell proliferation (25).

The above studies illustrate that using AMOs specific to oncogenic miRNA is a crucial approach to cancer therapy. Therefore, we constructed an AMOs-expression vector and investigated its supressive role in A549 cell proliferation. We found that miR-150 expression decreased and the apoptotic number of A549 cells increased after AMO-150-expression vector transfection. We also found that tumor volume became smaller and the weight was reduced in the AMO-150expression vector-treated group compared with the control group. Our results indicated that inhibition of miR-150 expression would be an effective option for lung cancer therapy.

\section{Materials and methods}

Vector construction. The polymerase III promoter (H1 promoter) was amplified by PCR from human genomic DNA and the $\mathrm{Tc}$ vector (containing the $\mathrm{H} 1$ promoter) was constructed as previously described (26). Then, the $\mathrm{H} 1$ promoter from the $\mathrm{Tc}$ vector was cloned into the pREP4 vector (Invitrogen) to form the PH vector. The antisense RNA targeting miRNA oligonucleotides were annealed and cloned into the $\mathrm{PH}$ vector using HindIII and AfII to designate the PR-ASO-150 vector. The forward (coding antisense RNA) sequence was 5 '- $\underline{\mathrm{AG}}$ CTCCCCACTGGTACAAGGGTTGGGAGTTTTTGGAA AGCTAGCGGTTTTCTGACTTCGGTCGGAAAACCC CTC-3'; the reverse sequence was 5'-TTAAGAGGGGTTTTC CGACCGAGTCAGAAAACCGCTAGCTTTCCAAAA ACTCCCAACCCTTGTACCAGTGGGG-3'. The forward coding sequence of a control fragment was 5 '-AGCTCCCCA GTACTTTTGTGTAGTCTTTTTTTGGAAAGCTAG CGGTTTTCTGACTTCGGTCGGAAAACCCCTC-3' and the reverse sequence was 5'-TTAAGAGGGGTTTTCCGACCG AGTCAGAAAACCGCTAGCTTTCCAAAAAAAGTACT ACACAAAAGTACTGGGG-3' (the underlined sequence in primer shows the restriction enzyme cut site).

Cell culture. A549 cells (human lung adenocarcinoma epithelial cells) were obtained from the Shanghai Institute of Cell Biology. A549 cells were maintained in F12 medium (Gibco) supplemented with $10 \%$ calf serum (Hyclone), $100 \mathrm{U} / \mathrm{ml}$ penicillin and $100 \mu \mathrm{g} / \mathrm{ml}$ streptomycin at $37^{\circ} \mathrm{C}$ with $5 \% \mathrm{CO}_{2}$.

Transient transfection was carried out using Lipofectamine 2000 according to the manufacturer's instructions (Invitrogen). The cells $\left(5 \times 10^{5}\right)$ were transfected with $1 \mu \mathrm{g}$ plasmid (PR-ASO150) and $2.5 \mu 1$ lipofectamine. The medium was changed after $8 \mathrm{~h}$. After transfection with PR-ASO-150 and incubation for $72 \mathrm{~h}$, samples were collected and the morphological changes of A549 cells were observed under an inverted microscope. All transfections were carried out in triplicate.

Detection of growth inhibition rate. After cells were tranfected with PR-ASO-150 for $72 \mathrm{~h}$, the MTT (3-(4,5-dimethylthiazol- 2-yl)-2,5-diphenyltetrazolium bromide) (Sigma) assay was carried as follows: $1 \times 10^{4}$ cells were cultured into each well of 96-well flat-bottom microtiter plates and $10 \mu \mathrm{l}$ MTT $(5 \mathrm{mg} / \mathrm{ml})$ were added $4 \mathrm{~h}$ before the end of incubation, the supernatant was removed and $100 \mu 1$ dimethyl sulfoxide (DMSO) were added to determine the OD value at $570 \mathrm{~nm}$ using an enzymelinked immunosorbent assay reader (ELX800). The growth inhibition rate was dectected according to the studies of Ren et al and O'Donovan et al $(27,28)$. The following formula was used: growth inhibition rate $=\left(\mathrm{OD}_{\text {control }}-\mathrm{OD}_{\text {sample }}\right) /$ $\mathrm{OD}_{\text {control }} \times 100(\%)$.

Detection of apoptotic cells by flow cytometry. For A549 cells, cells were trypsinized and gently washed with serumcontaining medium. The samples $\left(1 \times 10^{5}\right.$ cells $)$ were centrifuged for $5 \mathrm{~min}$ at $500 \mathrm{x} \mathrm{g}$ and the supernatant was discarded. Cells were resuspend in $500 \mu \mathrm{l}$ of $1 \mathrm{X}$ binding buffer. Propidium iodide $(5 \mu \mathrm{l})$ was added. The cells were incubated at room temperature for $5 \mathrm{~min}$ in the dark followed by analysis by flow cytometry (Beckman-Coulter, Inc., USA). The FL2 channel was chosen for detecting PI staining. The percentage of cells in the apoptotic phase was calculated.

miRNA detection by real-time PCR. miRNAs were isolated by a mirVana ${ }^{\mathrm{TM}}$ miRNA kit (Ambion) and poly (A) was added using a poly(A) polymerase (Ambion). The cDNA was synthesized by the RT primer: 5'-AACATGTACAGTCCATGGATG $\mathrm{d}(\mathrm{T})_{30} \mathrm{~N}(\mathrm{~A}, \mathrm{G}, \mathrm{C}$ or $\mathrm{T})-3^{\prime}$. The forward primer of miR-150 used to amplify the miRNAs was 5 '-tcccaacccttgtaccagt-3'. The reverse primer was 5'-AACATGTACAGTCCATGGATG-3'. Then qRT-PCRs were performed by SuperTaq polymerase (Takara) following the manufacturer's instructions. The expression of miRNAs was assessed using the RG3000 system (Corbett Research) with the Quantitect SYBR-Green kit (Qiagen). An initial denaturation at $95^{\circ} \mathrm{C}$ for $3 \mathrm{~min}$, followed by 40 cycles of $95^{\circ} \mathrm{C}$ for $30 \mathrm{sec}, 53^{\circ} \mathrm{C}$ annealing for $20 \mathrm{sec}$, and extension at $72^{\circ} \mathrm{C}$ for $20 \mathrm{sec}$ were performed. Fluorescence was detected at $585 \mathrm{~nm}$ at each extension step of $72^{\circ} \mathrm{C}$. The human $5 \mathrm{~S}$ rRNA served as the control.

p53 expression detected by Western blotting. A549 cells were lysed (lysis buffer, $0.15 \mathrm{M} \mathrm{NaCl} ; 5$ mM EDTA, pH 8.0; $1 \%$ Triton X-100; 10 mM Tris-HCl, pH 7.4; 100 mM PMSF). Protein $(35 \mu \mathrm{g})$ was loaded in each lane of a $12 \%$ SDS2 polyacrylamide gel, then transferred to a nitrocellulose membrane. The membranes were blocked with 5\% non-fat milk in TBST [50 mmol/l Tris- $\mathrm{HCl}$ (pH 7.6), $150 \mathrm{mmol} / \mathrm{l} \mathrm{NaCl}, 0.1 \%$ Tween20] for $2 \mathrm{~h}$ at room temperature. Subsequently, the membranes were incubated with a rabbit anti-p53 antibody (1:200, Boster Immunoleader) in blocking buffer at $4^{\circ} \mathrm{C}$ overnight. Following a wash with TBST, the membranes were incubated with a horseradish peroxidase-conjugated goat anti-rabbit secondary antibody (1:5000, Bei Jing Zhong Shan-Golden Bridge Technology Co., Ltd.) for $1 \mathrm{~h}$ at room temperature. The membranes were washed with TBST and protein bands were detected using the ECL/ECL plus kit (Boster Immunoleader).

A549 lung cancer xenografts. A549 cells were cultured and trypsinized, counted and subcutaneously injected into the lower back of 6-8-week old BALB/C-nu mice (nude mice, 


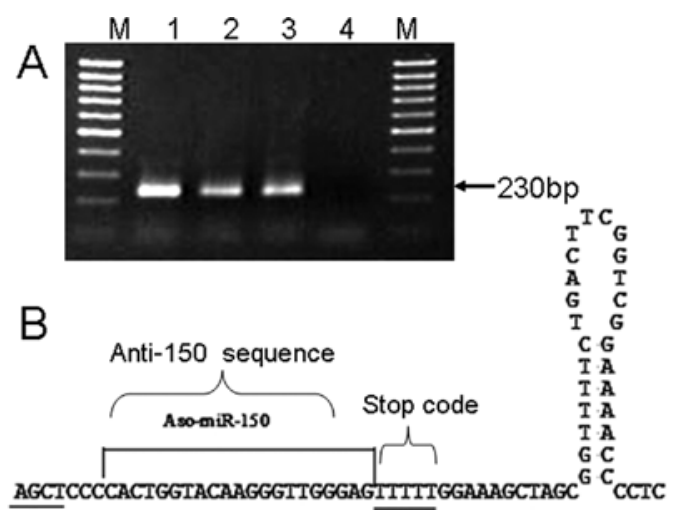

Figure 1. Construction of the PR-ASO-150 vector. (A) The H1 promoter was cloned by PCR. M, 100 bp marker. Lanes 1-3, H1 promoter (230 bp); lane 4, negative PCR control. (B) The sketch map of the PR-ASO-150 vector. The anti-miR-150 sequence is shown; the 'TTTTT' sequence is the stop code.

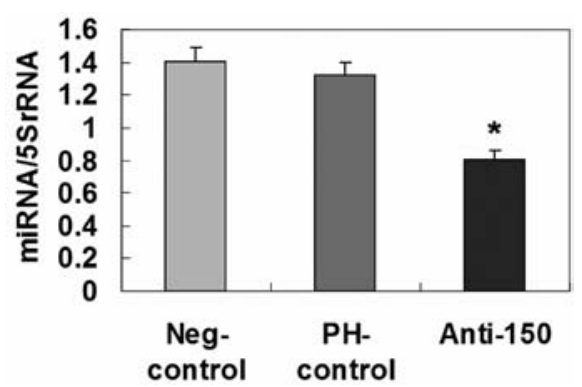

Figure 2. Inhibition of miR-150 expression by anti-miRNA vector (PR-ASO150). Real-time PCR results show that miR-150 expression is down-regulated after PR-ASO-150 treatment. Significant differences $(\mathrm{p}<0.05)$ are indicated with an asterisk.

HFK Bio-Technology) using $5 \times 10^{6}$ cells in $100 \mu$ F12 medium per injection. Once the mice developed palpable tumors, caliper measurements were taken daily and tumor volume was calculated using the formula: $\mathrm{V}=$ length $\mathrm{x}$ width ${ }^{2} / 2$ (length $>$ width). When tumors grew to a volume of $\sim 150 \mathrm{~mm}^{3}$,
$5 \mu \mathrm{g}$ PR-ASO-150 plasmid in $40 \mu \mathrm{l}$ PBS with the $12.5 \mu \mathrm{l}$ Lipofectamine (Invitrogen) were delivered intratumorally 3 times in 3-day intervals. Mice were sacrificed 5 days after the last treatment, and tumors were collected and prepared for subsequent analysis. All animal experiments were performed under an approved animal study protocol of the Binzhou Medical University.

Statistical analysis. The SAS software was used to analyze the significance of the results. The Student's t-test was used for intergroup comparison, and a p-value $<0.05$ was considered significant.

\section{Results}

Construction of the PR-ASO-150 vector. To drive the 20 -bp antisense RNA expression, the 200-bp polymerase III promoter (H1 promoter) was amplified by PCR from human genomic DNA (Fig. 1A). The H1 promoter was cloned into the PREP4 vector from the Tc plasmid to construct the PH vector. The $\mathrm{H} 1$ promoter sequence was verified using an automatic DNA sequencer (Biosune, Shanghai, China; data not shown). Then, the coding antisense RNA (specific to miR-150) was cloned downstream of the $\mathrm{H} 1$ promoter to form the PR-ASO-150 vector, in which the 'TTTTT' sequence was the stop code before the hairpin structure (Fig. 1B).

Inhibition of miR-150 expression in A549 cells. After cells were transfected with PR-ASO-150, the miRNAs were isolated and poly (A) was added. qRT-PCRs were performed with the SuperTaq Polymerase following the manufacturer's instructions. The results showed that miR-150 expression in A549 cells was lower in the PR-ASO-150-treated group than in the control group (Fig. 2).

Cell growth inhibition rate and apoptosis after PR-ASO-150 treatment. After PR-ASO-150 treatment, the number of A549 cells found alive was lower than that of the control groups
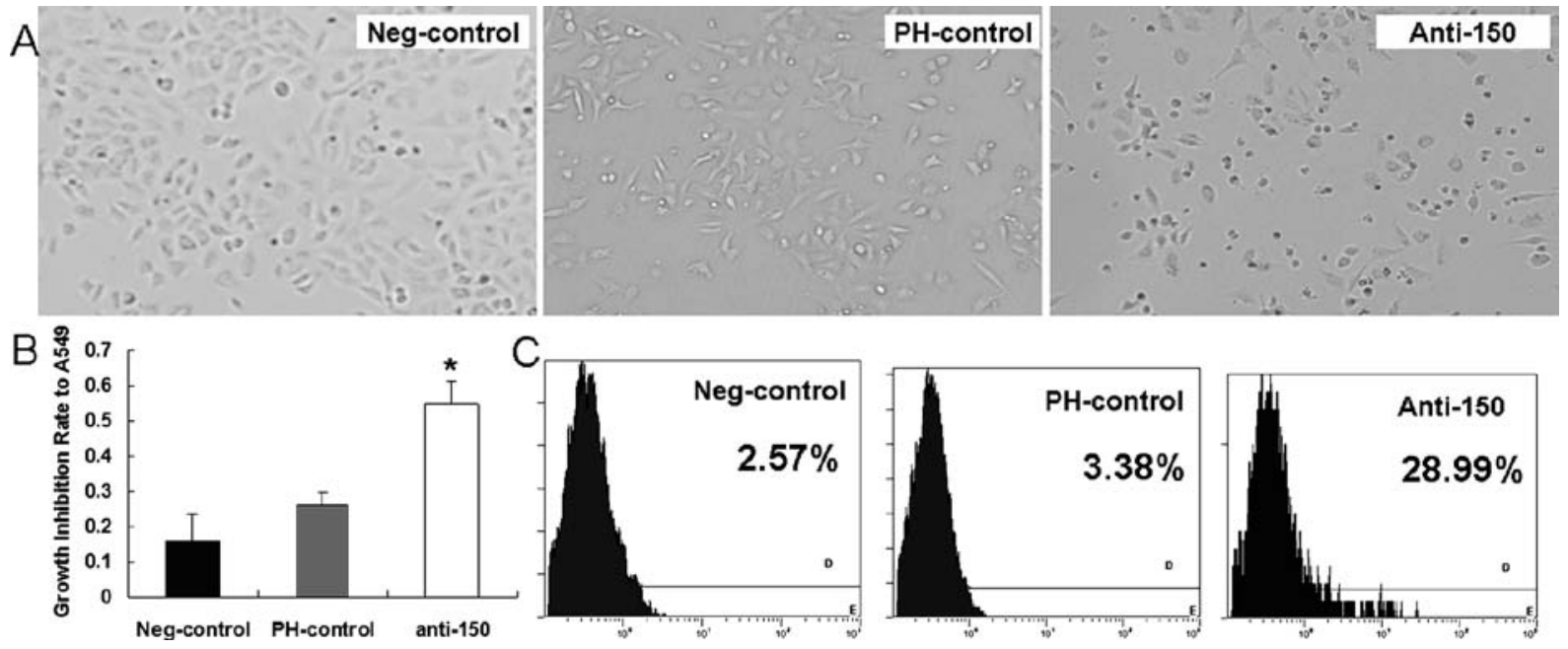

Figure 3. Cell growth inhibition rate and apoptosis analysis. (A) The number of live cells was analyzed by microscopy after PR-ASO-150 treatment, (x400). A549 cells growth was lower in the PR-ASO-150-treated group than in the control. (B) Growth-inhibitory rate of A549 cells analyzed by the MTT assay. The growth inhibition rate of A549 cells was $>50 \%$ in the PR-ASO-150-treated group compared with the control (p<0.05). (C) Flow cytometry analysis. The percentage of apoptotic cells was 3.38 and $28.99 \%$ in the control and PR-ASO-150 groups, respectively. 


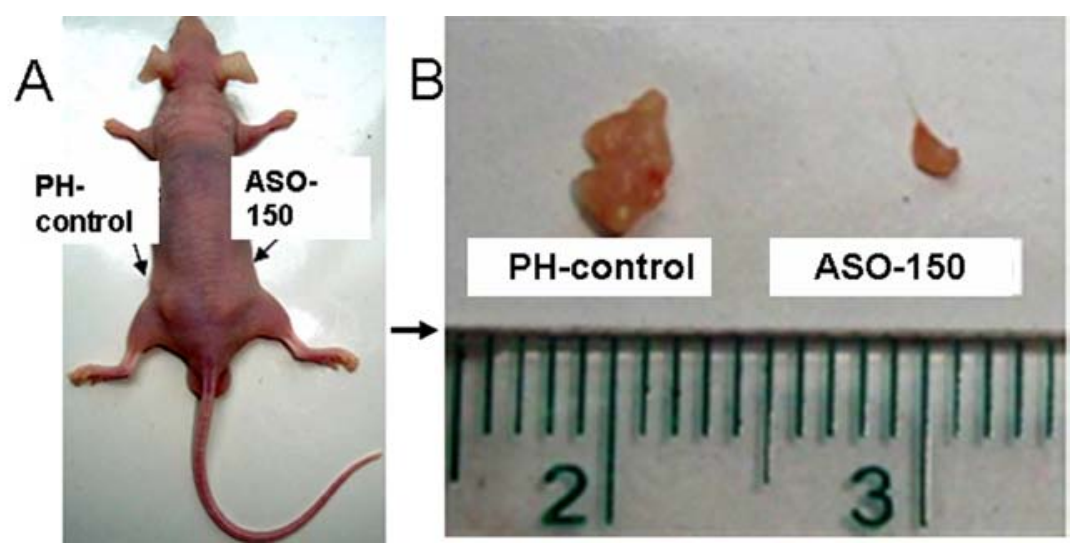

Figure 4. Growth inhibition of established A549 tumor xenografts by PR-ASO-150 vector. (A) Images of mice carrying A549 tumors after sacrifice on day 20. Subcutaneous tumors are indicated by arrows. (B) Tumor tissues were detected after operation. The results indicate that the tumor volume became smaller with PR-ASO-150 treatment.

Table I. Inhibition of tumor growth in mice by PR-ASO-150.

\begin{tabular}{lc}
\hline Groups $(\mathrm{n}=5)$ & Average $(\mathrm{g})$ \\
\hline PH-control & $0.01698 \pm 0.00118$ \\
ASO-150 & $0.00295 \pm 0.00139^{\mathrm{a}}$ \\
\hline
\end{tabular}

${ }^{\mathrm{a}} \mathrm{p}<0.05$ vs. PH-control.

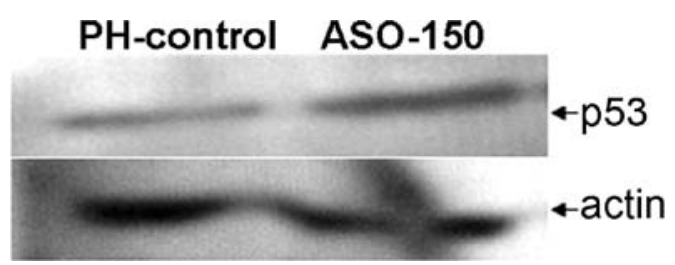

Figure 5. p53 expression in the tumor. p53 expression increased in the PR-ASO-150 treatment group compared with the control.

(Fig. 3A). In addition, the MTT assay was performed after the cells were exposure to PR-ASO-150 for $72 \mathrm{~h}$. Inhibition of the miR-150 expression by PR-ASO-150 resulted in a higher growth inhibition rate (Fig. 3B), indicating that PR-ASO-150 could inhibit A549 cell proliferation by regulating miR-150 expression. Detection of the apoptotic A549 cells by flow cytometry, demonstrated that the percentage of cells in the apoptotic phase was higher (28.99\%) in the PR-ASO-150 group compared to that in the control group (3.38\%) (Fig. 3C).

Inhibition of A549 cell growth in nude mice. After A549 cells were injected into the lower back of 6-8-week old BALB/C-nu mice, PR-ASO-150 plasmids were delivered intratumorally to observe the suppressive action of ASO-150 in A549 cells via regulation of miR-150 expression. The tumor volume and weight decreased by PR-ASO-150 treatment (Fig. 4, Table I). In a previous study, we found that p53 was a downstream factor negatively regulated by miR-150 (25). To investigate whether the changes of tumor volume and weight were relative to $\mathrm{p} 53$ expression, $\mathrm{p} 53$ expression in the tumor was assessed by
Western blotting. p53 expression increased after PR-ASO-150 treatment (Fig. 5), indicating that up-regulation of p53 is related to the suppression in tumor growth.

\section{Discussion}

Since their discovery in nematodes, the role of miRNAs in cancer has been extensively studied. Their frequent location at fragile sites, common break-points or regions of amplification or loss of heterozygosity reveal that miRNAs may play significant roles in human carcinogenesis (29). Anti-microRNA oligonucleotides (AMOs) specific to oncogenic miRNA is a potential approach to cancer therapy. In a previous study, miR-150 was found to act as an oncogene (25). Therefore, in this study, we constructed the PR-ASO-150 vector to express antisense RNA specific to miR-150 and found that it can inhibit miR-150 expression effectively in A549 cells. We also found that tumor volume decreased after PR-ASO-150 treatment compared to control vector treatment. The mechanism may be related to p53 expression, which was higher after PR-ASO-150 treatment compared with control group.

Changes in microRNA expression may be a downstream effect of potent oncogenes or tumor suppressors in the carcinogenesis process, such as the modulation of miR-34 by p53 (30). miR-21 is an oncogene, which plays a key role in programmed cell death resistance in cancer cells. miR-21 has been implicated in practical aspects of oncogenic life: the promotion of cell proliferation, invasion, metastasis, and evasion of apoptosis (31). miR-373 has been shown to regulate cell cycle progression by targeting PPP6C transcripts in vitro. The expression of miR-373 may also promote HCC cell proliferation (32).

In this study, we showed that A549 cell growth was suppressed by PR-ASO-150, which expressed antisense RNA specific to miR-150. The oncogenic role of miR-150 in cancer is also supported by the study of Zhou et al, in which miR-150 levels were observed to be higher in cancer than in normal cells, and increased expression of miR-150 in cancer epithelial cells was reported to decrease P2X7 mRNA by activation of miR-150 instability target sites located at the 3'-UTR-P2X7 (33). miR-150 has been found to be up-regulated in hepatoblastoma (34) in gastric cancer cell lines and tissues (35) and in colorectal cancer specimens with liver metastases (36). 
Ectopic expression of miR-150 can promote tumorigenesis and proliferation of gastric cancer cells.

Our studies as well as those of others indicate that miR-150 plays an important role in cancer cell growth progression and cellular metastasis. The discovery of miR-150 acting as an oncogene may open an unprecedented path in the targeted approach to cancer treatment. Antisense oligonucleotides, generally named anti-miRNAs, can bind directly to miRNAs and block their activity. With time, the AMOs in cells may be subjected to degradation by RNase in vivo and the inhibition to cell proliferation would disappear, though several chemical modifications to these oligonucleotides have been carried out to improve their efficacy (37). In order to express antisense RNA for a longer time, a vector-based anti-miRNA can express antisense RNA effectively, even constantly if it was intergated into genome. Therefore, we designed the anti-miR-150 expression vector (PR-ASO-150) regulated by using the $\mathrm{H} 1$ promoter and the 'TTTTT' sequence following a hairpin as the stop code. Our results demonstrate that miR-150 expression was effectively suppressed after PR-ASO-150 treatment of A549 cells.

In summary, we constructed an anti-miRNA vector (PR-ASO-150) to inhibit miR-150 expression in A549 cells. Tumor growth was suppressed in nude mice and p53 expression was higher after PR-ASO-150 treatment compared with the control group. Our study provides novel insight into cancer therapy by development of ASOs.

\section{Acknowledgements}

This study was supported by the NCET, 'Taishan scholar' position and National Natural Science Foundation (no. 30801324), the Shandong Science and Technology Committee (no. 2007BS03048, ZR2009CQ033, ZR2009CL005) and the Foundation of ShanDong Educational Committee of China (no. J09LF11). We also thank Xiuwen Wang and Lixia Zhang (Experimental Central Lab of Binzhou Medical University) for their help with the flow cytometry analyses.

\section{References}

1. Friedman RC, Farh KK, Burge CB and Bartel DP: Most mammalian mRNAs are conserved targets of microRNAs. Genome Res 19: 92-105, 2009.

2. Ambros V: MicroRNA pathways in flies and worms: growth, death, fat, stress, and timing. Cell 113: 673-676, 2003.

3. Garzon R, Calin GA and Croce CM: microRNAs in cancer. Annu Rev Med 60: 167-179, 2009.

4. Garzon R, Fabbri M, Cimmino A, Calin GA and Croce CM: MicroRNA expression and function in cancer. Trends Mol Med 12: 580-587, 2006.

5. Tong AW and Nemunaitis J: Modulation of microRNA activity in human cancer: a new paradigm for cancer gene therapy? Cancer Gene Ther 15: 341-355, 2008.

6. Garzon R, Liu S, Fabbri M, et al: MicroRNA-29b induces global DNA hypomethylation and tumor suppressor gene reexpression in acute myeloid leukemia by targeting directly DNMT3A and 3B and indirectly DNMT1. Blood 113: 6411-6418, 2009.

7. Marcucci G, Maharry K, Radmacher MD, et al: Prognostic signifcance of, and gene and microRNA expression signatures associated with, CEBPA mutations in cytogenetically normal acute myeloid leukemia with high-risk molecular features: a Cancer and Leukemia Group B Study. J Clin Oncol 26: 5078-5087, 2008.

8. Bueno MJ, Perez de Castro I, Gomez de Cedron M, et al: Genetic and epigenetic silencing of microRNA-203 enhances ABL1 and BCR-ABL1 oncogene expression. Cancer Cell 13: 496-506, 2008.
9. Eiring AM, Harb JG, Neviani P, et al: miR-328 functions as an RNA decoy to modulate hnRNPE2 regulation of mRNA translation in leukemic blasts. Cell 140: 652-665, 2010.

10. Venturini L, Battmer K, Castoldi M, et al: Expression of the miR-17-92 polycistron in chronic myeloid leukemia (CML) CD34+ cells. Blood 109: 4399-4405, 2007.

11. Bandi N and Vassella E: miR-34a and miR-15a/16 are co-regulated in non-small cell lung cancer and control cell cycle progression in a synergistic and Rb-dependent manner. Mol Cancer 10: 55 , 2011.

12. Jeong HC, Kim EK, Lee JH, Yoo HN and Kim JK: Aberrant expression of let-7a miRNA in the blood of non-small cell lung cancer patients. Mol Med Rep 4: 383-387, 2011.

13. Garcia AI, Buisson M, Bertrand P, Rimokh R, Rouleau E, Lopez BS, Lidereau R, Mikaélian I and Mazoyer S: Downregulation of BRCA1 expression by miR-146a and miR-146b-5p in triple negative sporadic breast cancers. EMBO Mol Med 3: 279-290, 2011

14. Gramantieri L, Ferracin M, Fornari F, Veronese A, Sabbioni S, Liu CG, Calin GA, Giovannini C, Ferrazzi E and Grazi GL: Cyclin G1 is a target of miR-122a, a microRNA frequently down-regulated in human hepatocellular carcinoma. Cancer Res 67: 6092-6099, 2007.

15. Gramantieri L, Fornari F, Ferracin M, Veronese A, Sabbioni S, Calin GA, Grazi GL, Croce CM, Bolondi L and Negrini M: MicroRNA221 targets Bmf in hepatocellular carcinoma and correlates with tumor multifocality. Clin Cancer Res 15: 5073-5081, 2009.

16. Favaro E, Ramachandran A, McCormick R, Gee H, Blancher C, Crosby M, Devlin C, Blick C, Buffa F and Li JL: MicroRNA-210 regulates mitochondrial free radical response to hypoxia and Krebs cycle in cancer cells by targeting iron sulfur cluster protein ISCU. PLoS One 5: e10345, 2010.

17. Cascio S, D'Andrea A, Ferla R, Surmacz E, Gulotta E, Amodeo V, Bazan V, Gebbia N and Russo A: miR-20b modulates VEGF expression by targeting HIF-1 $\alpha$ and STAT3 in MCF-7 breast cancer cells. J Cell Physiol 224: 242-249, 2010.

18. Weiler J, Hunziker J and Hall J: Anti-microRNA oligonucleotides (AMOs): ammunition to target microRNAs implicated in human disease? Gene Ther 13: 496-502, 2006.

19. Baker BF, Lot SS, Condon TP, Cheng-Flournoy S, Lesnik EA, Sasmor HM and Bennett CF: 2'-O-(2-Methoxy)ethyl-modified anti-intercellular adhesion molecule 1 (ICAM-1) oligonucleotides selectively increase the ICAM-1 mRNA level and inhibit formation of the ICAM-1 translation initiation complex in human umbilical vein endothelial cells. J Biol Chem 272: 11994-12000, 1997.

20. Esau C, Davis S, Murray SF, Yu XX, Pandey SK, Pear M, Watts L, Booten SL, Graham M and McKay R: miR-122 regulation of lipid metabolism revealed by in vivo antisense targeting. Cell Metab 3: 87-98, 2006.

21. Volinia S, Calin GA, Liu CG, Ambs S, Cimmino A, Petrocca F, Visone R, Iorio M, Roldo C and Ferracin M: A microRNA expression signature of human solid tumors defines cancer gene targets. Proc Natl Acad Sci USA 103: 2257-2261, 2006.

22. Iorio MV, Ferracin M, Liu CG, Veronese A, Spizzo R, Sabbioni S, Magri E, Pedriali M, Fabbri M and Campiglio M: MicroRNA gene expression deregulation in human breast cancer. Cancer Res 65: 7065-7070, 2005.

23. Lu Y, Xiao J, Lin H, Bai Y, Luo X, Wang Z and Yang B: A single anti-microRNA antisense oligodeoxyribonucleotide (AMO) targeting multiple microRNAs offers an improved approach for microRNA interference. Nucleic Acids Res 37: e24, 2009.

24. Fei J, Lan F, Guo M, Li Y and Liu Y: Inhibitory effects of antimiRNA oligonucleotides (AMOs) on A549 cell growth. J Drug Target 16: 688-693, 2008.

25. Wang PY, Li YJ, Zhang S, Li ZL, Yue Z, Xie N and Xie SY: Regulating A549 cells growth by ASO inhibiting miRNA expression. Mol Cell Biochem 339: 163-171, 2010.

26. Xie SY, Zhang JZ, Huang SZ, Sun D, Ren ZR and Zeng YT: Suppression of eGFP expression in erythroid-specific transgenic mice by siRNA. Blood Cells Mol Dis 34: 220-225, 2005.

27. Ren H, Tian L, Gu Q and Zhu W: Secalonic acid D: a cytotoxic constituent from marine lichen-derived fungus Gliocladium $s p$. T31. Arch Pharm Res 29: 59-63, 2006.

28. O'Donovan DJ, Katkin JP, Tamura T, Smith CV and Welty SE: Attenuation of hyperoxia-induced growth inhibition in H441 cells by gene transfer of mitochondrially targeted glutathione reductase. Am J Respir Cell Mol Biol 22: 732-738, 2000.

29. Budhu A, Ji J and Wang XW: The clinical potential of microRNAs. J Hematol Oncol 3: 37, 2010. 
30. Chang TC, Wentzel EA, Kent OA, et al: Transactivation of miR-34a by $\mathrm{p} 53$ broadly influences gene expression and promotes apoptosis. Mol Cell 26: 745-752, 2007.

31. Buscaglia LE and Li Y: Apoptosis and the target genes of microRNA-21. Chin J Cancer 30: 371-380, 2011.

32. Wu N, Liu X, Xu X, Fan X, Liu M, Li X, Zhong Q and Tang H: MicroRNA-373, a new regulator of 6 , functions as an oncogene in hepatocellular protein phosphatase carcinoma. FEBS J: April 11, 2011 (Epub ahead of print), doi: 10.1111/j.1742-4658. 2011.08120.x.

33. Zhou L, Qi X, Potashkin JA, Abdul-Karim FW and Gorodeski GI: MicroRNAs miR-186 and miR-150 down-regulate expression of the pro-apoptotic purinergic $\mathrm{P} 2 \mathrm{X} 7$ receptor by activation of instability sites at the 3'-untranslated region of the gene that decrease steady-state levels of the transcript. J Biol Chem 283 : 28274-28286, 2008
34. Magrelli A, Azzalin G, Salvatore M, Viganotti M, Tosto F, Colombo T, Devito R, Di Masi A, Antoccia A, Lorenzetti S, Maranghi F, Mantovani A, Tanzarella C, Macino G and Taruscio D: Altered microRNA expression patterns in hepatoblastoma patients. Transl Oncol 2: 157-163, 2009.

35. Wu Q, Jin H, Yang Z, Luo G, Lu Y, Li K, Ren G, Su T, Pan Y, Feng B, Xue Z, Wang X and Fan D: MiR-150 promotes gastric cancer proliferation by negatively regulating the pro-apoptotic gene EGR2. Biochem Biophys Res Commun 392: 340-345, 2010.

36. Lin M, Chen W, Huang J, Gao H, Ye Y, Song Z and Shen X: MicroRNA expression profiles in human colorectal cancers with liver metastases. Oncol Rep 25: 739-747, 2010.

37. Meister G, Landthaler M, Dorsett Y and Tuschl T: Sequence specific inhibition of microRNA- and siRNA-induced RNA silencing. RNA 10: 544-550, 2004. 